\title{
BANACH SPACES OF FUNCTIONS ANALYTIC IN A POLYDISC
}

\author{
LEON M. HALL \\ Department of Mathematics and Statistics \\ University of Missouri-Rolla \\ Rolla, Missouri 65401 U.S.A.
}

(Received March 8, 1985)

ABSTRACT. This paper is concerned with functions of several complex variables analytic in the unit olydisc. Certain Banach spaces to which these functions might belong are defined and some relationships between them are developed. The space of linear functionals for the Banach space of functions analytic in the open unit polydisc and continuous on the unit torus is then described in terms of analytic functions using an extension of the hadamard product.

KEY WORDS AND MHASES. Banach space, linear functional, analytic function. 1330 VIATHEMATICE SUBJECT CLAS:IFICATION CODE. $46 \mathrm{~W} 15$

3. INTRODUCTION.

In 1950 A. E. Taylor [1] studied Banach spaces of functions analytic in the unit disc. One of his principal results was a representation of linear functionals in terms of functions analytic in the unit disc. In this paper, the results of Taylor are extended to functions analytic in the unit polydisc in $\mathrm{n}$-dimensional complex space. The goal is the representation theorem for linear functions, Theorem 4.5, in which the functionils are expressed in terms of a Hadamard product. Taylor's results have proved to be very useful in work involving certain singular linear differential operators. His representation theorem for linear functionals was a key part of the work of Grimm and Hall in [2], and of subsequent work of Hall in [3] and [4].

The following notation will be used extensively:

$\mathrm{U}=$ open unit disc in the complex plane $\mathrm{C}$,

$T=$ unit circle in $\mathrm{C}$,

$T_{\varepsilon}=\left\{z \varepsilon C: \mid z_{1}=1-\varepsilon\right\}, 0<\varepsilon<1$,

+ - ronnegative integers,

$U^{n}=U \times U \times \ldots \times U$, ( $n$ copies of $U$ ) the unit polydisc in $c^{n}$,

$T^{n}=T \times T \times \ldots \times T$, ( $n$ copies of $T$ ) the unit torus in $C^{n}$,

$T_{\varepsilon}^{\mathrm{n}}=T_{\varepsilon} \times T_{\varepsilon} \times \ldots \times T_{\varepsilon^{\prime}}$ (n coples of $T_{\varepsilon}$ ),

$z_{+}^{n}=z_{+} \times z_{+} \times \ldots \times z_{+}\left(n\right.$ copies of $\left.z_{+}\right)$. 
If $z=\left(z_{1}, z_{2}, \ldots, z_{n}\right)$ and $w=\left(w_{1}, w_{2}, \ldots, w_{n}\right)$ are in $c^{n}$, define $z w$ by $z w=\left(z_{1} w_{1}, z_{2} w_{2}\right.$, $\left.\ldots, z_{n} w_{n}\right)$. Further, if $z \in C^{n}$ and $\alpha=\left(\alpha_{1}, \alpha_{2}, \ldots, \alpha_{n}\right) \varepsilon z_{+}^{n}$ define $z^{\alpha}$ by $z^{\alpha}=$ $z_{1}{ }_{1} z_{2}{ }^{\alpha} \ldots \ldots z_{n}{ }^{n}$ and $|\alpha|$ by $|\alpha|=\alpha_{1}+\alpha_{2}+\ldots+\alpha_{n}$. Denote by $A^{n}$ the class of functions on $C^{n}$ which are analytic in $U^{n}$ and define the function $u_{\alpha} \in A^{n}$, for each $\alpha \varepsilon Z_{+^{\prime}}^{n}$ by $u_{\alpha}(z)=z^{\alpha}$. Also define the operators $u_{x}: A^{n} \rightarrow A^{n}$ and $T_{w} A^{n} \rightarrow A^{n}$ by

$$
\begin{aligned}
& u_{x} f=g, \text { where } x=\left(x_{1}, x_{2}, \ldots, x_{n}\right) \in \mathbb{R}^{n}, \text { and } \\
& g(z)=f\left(z e^{1 x}\right) \equiv f\left(z_{1} e^{1 x_{1}}, z_{2} e^{i x_{2}}, \ldots, z_{n} e^{i x_{n}}\right), \\
& T_{w} f=g, \text { where } w \varepsilon \frac{U^{n}}{} \text {, and } g(z)=f(w z) .
\end{aligned}
$$

The operators $U_{x}$ and $T_{w}$ are easily seen to be linear.

If $f \in A^{n}$ then $f$ has the power series expansion

$$
f(z)=\sum_{\alpha \varepsilon z_{+}^{n}} f_{\alpha} z^{\alpha} .
$$

Denote $f_{\alpha}$ by $\gamma_{\alpha}(f)$ where $\gamma_{\alpha}$ is the functional defined by

$$
\gamma_{\alpha}(f)=\left.\left(\alpha_{1} ! \alpha_{2} ! \ldots \alpha_{n} !\right)^{-1} \frac{\partial^{|\alpha|_{f}}}{\partial z_{1} \ldots \partial_{n}{ }_{n}}\right|_{z=(0, \ldots, 0) .}
$$

If $f$ and $g$ are in $A^{\mathrm{n}}$ with power series

$$
f(z)=\sum_{\alpha \varepsilon z_{+}^{n}} f_{\alpha} z^{\alpha} \text { and } g(z)=\sum_{\alpha \varepsilon z_{+}^{n}} g_{\alpha} z^{\alpha},
$$

define the Hadamard product of $f$ and $g$ by

$$
H(f, g ; z)=\sum_{\alpha \varepsilon z_{+}^{n}} f{ }_{\alpha}^{g} z^{\alpha} .
$$

$H(f, g ; z)$ is clearly in $A^{n}$, and the following also can be proved:

$$
\begin{aligned}
& \gamma_{\alpha}\left(T_{w} f\right)=w^{\alpha} \gamma_{\alpha}(f), \\
& H(f, g ; z)=H(g, f ; z), \\
& H(a f+b g, h ; z)=a H(f, h ; z)+b H(g, h ; z), \\
& H\left(T_{w} f, g ; z\right)=H(f, g ; w z), \\
H(f, g ; z)= & \left(\frac{1}{2 \pi i}\right)^{n} \int_{\tau_{\varepsilon_{1}} x_{\ldots} . . \times T} f\left(\xi_{\varepsilon_{n}}, \ldots, \xi_{n}\right) g\left(\frac{z_{1}}{\xi_{1}}, \ldots, \frac{z_{n}}{\xi_{n}}\right) \frac{d \xi_{1}}{\xi_{1}} \ldots \frac{d \xi_{n}}{\xi_{n}},
\end{aligned}
$$

where $\left|z_{i}\right|<\left|\xi_{i}\right|$ for $1=1, \ldots, n$.

Equation (1.20) can also take the form

$$
H(f, g ; z)=\left(\frac{1}{2 \pi}\right)^{n} \int_{0}^{2 \pi} \ldots \int_{0}^{2 \pi} f\left(r_{1} e^{i \theta_{1}}, \ldots, r_{n} e^{i \theta_{n}}\right) g\left(\frac{z}{1}_{r_{1}} e^{-i \theta} 1, \ldots, \frac{z_{n}}{r_{n}}{ }^{-i \theta}{ }_{n}\right) d \theta_{1} \ldots d \theta_{n}
$$

where $r_{i}=1-\varepsilon_{i}$ and $z \varepsilon U^{n}$. 
2 SPACES OF TYPE $A_{k}$.

Let $B$ be a nontrivial complex Banach space, each element of which belongs to $A^{n}$. Such a space will be called a space of type A. B may also have one or more of the following ryoperties.

$P_{1}$ : There exists a constant A such that $\left|\gamma_{\alpha}(f)\right| \leq A|| f||$ if $f \varepsilon$ B and $\alpha \varepsilon Z_{+}^{\text {n }}$. The least such constant wlll be denoted $A_{1}(B)$.

$P_{2}: u_{\alpha} \in B$ for all $\alpha \in Z_{+}^{n}$. There exists a constant $A$ such that $\left\|u_{\alpha}\right\| \leq A$ for all $\alpha$. The least such constant will be denoted $A_{2}(B)$.

$P_{3}: U_{x} f \in B$ if $f \in B$ and $x$ is a real $n$-tuple. Also ||$u_{x} f||=|| f||$.

$\mathrm{P}_{4}: T_{r} \in \mathrm{B}$ if $\mathrm{f} \varepsilon \mathrm{B}$ and $r=\left(r_{1}, r_{2}, \ldots, r_{n}\right)$ with $0 \leq r_{i}<1$. There exists a constant A such that ||$T_{r} f|| \leq A|| f||$. The least such constant will be denoted $\mathrm{A}_{4}(\mathrm{~B})$.

$B$ will be called a space of type $A_{k}$ if $B$ is of type $A$ and also satisfies axioms $\mathrm{P}_{1}, \ldots, \mathrm{P}_{\mathrm{k}}$.

Let $B^{*}$ denote the space of continuous linear functionals on $B$. Then by $P_{1}$, $\gamma_{n} \in B^{*}$ and $1 t$ can be shown that

$$
\begin{aligned}
& A_{1}(B)=\sup _{\alpha}|| \gamma_{\alpha}|| \text {, and } \\
& A_{2}(B)=\sup _{\alpha}|| u_{\alpha}|| \text {. }
\end{aligned}
$$

Other relations satisfied by the constants $A_{k}(B)$ are

$$
\begin{gathered}
A_{4}(B)=\sup _{r}|| T_{r} \|, \\
1 \leq A_{1}(B) A_{2}(B), \\
1 \leq A_{4}(B)
\end{gathered}
$$

Let $B$ be a space of type $A_{1}$. For any fixed $g \varepsilon A^{n}$ and $z \varepsilon U^{n}$ define

$$
N(g ; z)=\sup _{|| f||=1}|H(f, g ; z)| .
$$

The following are clear from the properties of the Hadamard product.

$$
\begin{gathered}
\mathrm{N}(g+h ; z) \leq N(g ; z)+N(h ; z) \\
N(a g ; z)=|a| N(g ; z) \\
N\left(T_{w} g ; z\right)=N(g ; w z),|w|<1 .
\end{gathered}
$$

THEOREM 2.1. Let $B$ be a space of type $A_{3}$. Then the function $N(g ; z)$ has the properties:

$$
\begin{aligned}
& \mathrm{N}(g, z)=\mathrm{N}(g, \hat{z}) \text {, where } g \in A^{\mathrm{n}}, \mathrm{z} \varepsilon \mathrm{U}^{\mathrm{n}} \text { and } \hat{z}=\left(\left|\mathrm{z}_{1}\right|,\left|z_{2}\right|, \ldots,\left|\mathrm{z}_{n}\right|\right), \\
& \mathrm{N}\left(g ;\left(r_{1}, \ldots, r_{n}\right)\right) \text { is a nondecreasing function of each } r_{i}, i=1, \ldots, \mathrm{m}, \\
& \text { where } 0 \leq r_{i}<1 \text {, } \\
& \mathrm{N}\left(g ;\left(r_{1}, \ldots, r_{n}\right)\right)=0 \text { for all }\left(r_{1}, \ldots, r_{n}\right) \text { if and only if } g \equiv 0 .
\end{aligned}
$$


PROOF. Let $M$ denote the set of all nonzero elements of $B$ and define, for $f \in M$

$$
M(f ; z)=\frac{H(f, g ; z)}{\|f\|}
$$

where $g$ is fixed in $A^{m}$, Also define

$$
M(z)=\sup _{f \in M}|M(f ; z)|
$$

Observe that $M(z)=N(g ; z)$ and also, for $x \in R^{n}$ and $z \varepsilon U^{n}$

$$
M\left(u_{x} f z\right)=M\left(f ; z e^{i x}\right)
$$

Let $z \in U^{\mathrm{n}}$ be fixed. Then

$$
\begin{aligned}
|M(f ; z)| & =\left|H\left(\frac{f}{\prod f} \mid, g ; z\right)\right| \\
& =\frac{1}{\prod f \mid}|H(f, g ; z)| \\
& \leq \prod^{1} \prod_{\alpha}^{\Sigma}\left|f_{\alpha}\right|\left|g_{\alpha}\right|\left|z^{\alpha}\right| \\
& \leq \prod_{\alpha}^{1} \prod_{\alpha}^{\sum A_{1}(B)|| f||}\left|g_{\alpha}\right|\left|z^{\alpha}\right| \\
& =A_{1} \text { (B) } \sum_{\alpha}\left|g_{\alpha}\right|\left|z^{\alpha}\right| .
\end{aligned}
$$

Hence, for fixed $z \varepsilon U^{n},|M(f ; z)|$ is bounded as $f$ varies over $M$.

Now for $x \in R^{n}, U_{x} f$ varies over all of $M$ as $f$ varies over all of $M$ since $f=$ $u_{x}\left(u_{x} f\right)$, and so by $(2.15)$ we get $M(z)=M(\hat{z})$ if $x$ is chosen carefully, and $(2.10)$ is proved.

Next, let $R=\left(R_{1}, \ldots, R_{i}, \ldots, R_{n}\right)$ and $r=\left(r_{1}, \ldots, r_{i}, \ldots, r_{n}\right)$ with $0 \leq R_{k}=r_{k}<1$ for $k=1, \ldots, n, k \neq i$ and $0 \leq r_{i}<R_{i}<1$. Also suppose $f \in M$. Then let $z_{R}$ and $z_{r}$ be the points on the tori $\left\{z:\left|z_{k}\right|=R_{k}\right\}$ and $\left\{z:\left|z_{k}\right|=r_{k}\right\}$, respectively, at which $|M(f ; z)|$ assumes its maximum value. The maximum principle for polydisc functions now yields

$$
\left|M\left(f ; z_{r}\right)\right| \leq\left|M\left(f ; z_{R}\right)\right|
$$

But $\left|M\left(f ; z_{R}\right)\right| \leq M\left(z_{R}\right)=M\left(\hat{z}_{R}\right)=M(R)$ and so

$$
|M(f ; r)| \leq\left|M\left(f ; z_{r}\right)\right| \leq M(R),
$$

which implies $M(r) \leq M(R)$, and $(2.11)$ is proved.

Finally, if $g \equiv 0, N(g, r) \equiv 0$ for all $r$. If $g \neq 0$, then $\gamma_{\alpha}(g) \neq 0$ for some $\alpha$. Let $\mathrm{f}=\mathrm{u}_{\alpha}$ for this $\alpha$. Then $\mathrm{H}(\mathrm{f}, \mathrm{g} ; \mathrm{r})=\gamma_{\alpha}(\mathrm{g}) \mathrm{r}_{1}{ }^{\alpha_{1}} \ldots \mathrm{r}_{\mathrm{n}}^{\alpha_{\mathrm{n}}}$ which is nonzero if $r_{\mathrm{k}} \neq 0$, $k=1, \ldots, n$, and the proof is complete.

3. THE SPACES $B^{\prime}$ AND $B^{\circ}$.

Suppose $B$ is a space of type $A_{3}$. Two related spaces which will be used later in characterizing $B^{*}$ will be defined as follows:

$B^{\prime}=\left\{F \varepsilon A^{n}: N(F ; r)\right.$ is uniformly bounded in $\left.r, 0 \leq r<<, k=1, \ldots, n\right\}$, 


$$
B^{O}=\left\{F \in A^{n}: \lim _{r \rightarrow(1, \ldots, 1)} H(f, F ; r) \text { exists for each } f \varepsilon B\right\} \text {. }
$$

For $F \in B^{l}$ define $N(F)=\sup \left\{N(f ; r): 0 \leq r_{k}<1, k=1, \ldots, n\right\}$. By (2.11) we have

$$
N(F)=\lim _{r(1, \ldots, 1)} N(F ; r)
$$

THEOREM 3.1. If $B$ is a space of type $A_{3}$ then $B^{\prime}$ is a Banach space with norm $N(F)$. PROOF. From the properties of the Hadamard product and $N(F ; r)$, and from theorem 2. $1, B^{\prime}$ is clearly a normed linear space with norm $N(F)$. To prove completeness proceed as follows.

Let $\left\{F_{j}\right\}$ be a Cauchy sequence in $B^{\prime}$. Then there is a $J>0$ such that if $j>J$, $N\left(F_{j}\right)-N\left(F_{J}\right)<1$, and $N\left(F_{j}\right)$ is bounded by, say, $E_{\text {。 }}$ Let $0 \leq r_{k}<1$ for $k=1, \ldots, n$ and consider, for a $\varepsilon z_{+}^{m}$,

$$
\begin{aligned}
\left|\gamma_{\alpha}\left(F_{j}-F_{m}\right) r^{\alpha}\right| & =H\left(u_{\alpha}, F_{j}-F_{m} ; r\right) \mid \\
& \leq|| u_{\alpha}|| N\left(F_{j}-F_{m}\right) \leq A_{2}(B) N\left(F_{j}-F_{m}\right) .
\end{aligned}
$$

Thus, $\left\{\gamma_{\alpha}\left(F_{J}\right)\right\}$ is also a Cauchy sequence for each $\alpha \varepsilon z_{+}^{n}$. Now define $a_{\alpha}=\lim _{j \rightarrow \infty} \gamma_{\alpha}\left(F_{j}\right)$ and note that convergence is uniform in $\alpha$ and that the sequence $\left\{a_{\alpha}\right\}, \alpha \varepsilon z_{+}^{n}$, is bounded. Using the above argument with $F_{m}=0$ we obtain $\left|\gamma_{\alpha}\left(F_{j}\right) r^{\alpha}\right| \leq A_{2}(B) E$ which yields $\left|a_{\alpha}\right| \leq A_{2}(B) E$. Next define

$$
F(z)=\sum_{\alpha \in z_{+}^{n}} a_{\alpha} z^{\alpha}
$$

Clearly $F \in A$, and it will be shown that $F \in B^{\prime}$ and $\lim _{j \rightarrow \infty} F_{j}=F$.

Consider, for fixed $r$, and $f \in A$,

$$
|H(f, F ; r)-H(f, F ; r)| \leq \sum_{\alpha}\left|\gamma_{\alpha}(f) r^{\alpha} \sup _{\alpha}\right| \gamma_{\alpha}\left(F_{j}\right)-a_{\alpha} \mid \text {. }
$$

Since $\lim _{j \rightarrow \infty}\left[\gamma_{\alpha}\left(F_{j}\right)-a_{\alpha}\right]=0$ uniformly in $\alpha$,

$$
\lim _{j \rightarrow \infty} H\left(f, F_{j} ; r\right)=H(f, F ; r) .
$$

Further, for $f \in B$

$$
\left|H\left(f, G_{j} ; r\right)\right| \leq|| f|| N\left(F_{j}\right) \leq E|| f||
$$

whrch implies $|H(f, F ; r)| \leq E|| f||$ or $N(F ; r) \leq E$. Hence, $F \varepsilon B^{\prime}$.

Finally, for $\varepsilon>0$, choose $j_{0}(\varepsilon)$ such that if $j, m \geq j_{0}(\varepsilon)$ then $N\left(F_{j}-F_{m}\right)<\varepsilon$. For f $\varepsilon B(3.5)$ yields

$$
\left|H\left(f, F_{j}-F_{m} ; r\right)\right| \leq N\left(F_{j}-F_{m}\right)|| f||<\varepsilon|| f|| \text {. }
$$

As $m \rightarrow \infty$ (3.4) yuelds $\left|H\left(f, F_{j}-F ; r\right)\right| \leq \varepsilon|| f||$, if $j \geq j_{0}(\varepsilon)$, which means $N(F,-F) \leq \varepsilon$ and the proof is complete.

THEOREM 3.2. If $B$ is a Banach space of type $A_{3}$ then $B^{0}$ is a linear subset of $B^{\prime}$ and is a Banach space of type $A_{4}$ with norm $N(F)$. 
PROOF: Ciearly $B^{0}$ is a linear subset of $A$. For $f \varepsilon B$ and $F \varepsilon B^{0}$ let $\psi_{r}(f)=$ $H(f, F ; r)$ define a functional of $f$ over $B$. Then

$$
\sup _{\| f||=1}\left|\psi_{r}(f)\right|=N(F ; r) \text {, }
$$

and since $F \in B^{0}$ then $N(f ; r)$ is bounded as a function of $r$ by the uniform boundedness principle. Thus, $B^{0}$ is a linear subset of $B^{\prime}$ 。

In order to show that $B^{0}$ is closed in $B^{\prime}$, let $\left\{F_{j}\right\} \subset B^{0}$ and $F \varepsilon B^{\prime}$ be such that $\lim _{j \rightarrow \infty} N\left(F_{j}-F\right)=0 \quad$ For $f \in B$

$$
\begin{aligned}
\mid H(f, F ; r)-H(f, F & ; \rho)|\leq| H\left(f, F-F_{j} ; r\right)|+| H\left(f, F_{j} ; r\right)-H\left(f, F_{j} ; \rho\right)|+| H\left(f, F_{j}-F_{j} ; \rho\right) \mid \\
& \leq 2 N\left(F_{j}-F\right)|| f||+\left|H\left(f, F_{j} ; r\right)-H\left(f, F_{j} ; \rho\right)\right| 。
\end{aligned}
$$

For $f \varepsilon B$ flxed and $\varepsilon>0$ choose $j$ large enough so that $2 N\left(F_{j}-F\right)|| f||<\varepsilon / 2$ and choose $r$ and $\rho$ close enough to $(1,1, \ldots, 1)$ so that $\mid H\left(f, F_{j} ; r\right)-H\left(f, F_{j} ; \rho\right)<\varepsilon / 2$. Therefore $\lim _{r, \rho \rightarrow(l, \ldots, l)}|H(f, F ; r)-H(f, F ; \rho)|=0$ and $F \varepsilon B^{0}$.

4. REPRESENTATION OF LINEAR FUNCTIONALS.

In this section a series of results which culminate in a representation of the space $B^{*}$ and 1 ts relationshıp to the spaces $B^{\prime}$ and $B^{0}$ will be proved.

THEOREM 4.1. Let $B$ be a Banach space of type $A_{4}$. If $\gamma \varepsilon B^{*}$ let

$$
G(z)=\sum_{\alpha \varepsilon Z_{+}^{n}} \gamma\left(u_{\alpha}\right) z^{\alpha}, z \varepsilon U^{n} \text {. }
$$

Then $G \in B^{\prime}$ and ||$G||=N(G) \leq A_{4}(B)|| \gamma||$.

PROOF: $G \in A^{n}$ since $\left|\gamma\left(u_{\alpha}\right)\right| \leq\|\gamma\| A_{2}(B)$. Also, $\gamma(u)=\gamma_{\alpha}(G)=G_{\alpha}$. Let f $\varepsilon A^{n}$ and $w \in U^{n}$. Then $T_{w} f \in B$ and

$$
\gamma\left(T_{w} f\right)=\sum_{\alpha} f_{\alpha} \gamma\left(z^{\alpha}\right) w^{\alpha}=\sum_{\alpha} f_{\alpha} G_{\alpha} w^{\alpha}=H(f, G ; w) .
$$

If $f \in B$ then

$$
|H(f, G ; r)|=\left|\gamma\left(T_{r} f\right)\right| \leq A_{4}(B)|| \gamma|||| f|| \text {. }
$$

Hence $N(G ; r)=\sup _{|f| \mid=1}|H(f, G ; r)| \leq A_{4}(B)|| r||$ and $G \varepsilon B^{\prime}$. Also, ||$G||=N(G)=\sup _{r} N(G ; r) \leq A_{4}(B)\|\gamma\|$.

The passage from $\gamma$ to $G$ given by $(4.1)$ defines an operator $\Gamma: B^{\star} \rightarrow B^{\prime}$ where $\Gamma(\gamma)=G$.

THEOREM 4.2. Let $B$ be a Banach space of type $A_{4}$. Then $\Gamma$ is a linear operator and ||$\Gamma||=A_{4}(B)$. $\Gamma$ has an inverse if and only if the linear subspace of $B$ spanned by $\left\{u_{\alpha}\right\}, \alpha \varepsilon Z_{+}^{n}$, is dense in $B$.

PROOF, By Theorem $4.1 \Gamma$ is linear and ||$\Gamma|| \leq A_{4}(B)$. By (4.2)

$$
\left|\gamma\left(T_{f}^{f}\right)\right| \leq\|f\|\|G\| \leq\|f\|\|\Gamma\|\|\gamma\| \text {. }
$$

Now choose $\gamma$ so that $\| \gamma||=1$ and $\left|\gamma\left(T_{r} f\right)\right|=|| T_{r} f||$ so that $\left\|T_{r} f|| \leq\right\| f \||| \Gamma||$. 
Then $\left\|T_{r}\right\| \leq\|\Gamma\|$ and $A_{4}(B) \leq \| \Gamma||$. Hence ||$\Gamma||=A_{4}(B)$.

Suppose $\Gamma(\gamma)=0$. Then $\gamma\left(u_{\alpha}\right)=0$ for all $\alpha \varepsilon Z_{+}^{n}$ and hence saying that $\Gamma^{-1}$ exists if anc only if $\gamma\left(u_{\alpha}\right)=0$ for all $\alpha \varepsilon Z_{+}^{n}$ is equivalent to saying $\gamma=0$. Thus $\Gamma^{-1}$ exists If and only if $\left\{u_{\alpha}\right\}$ is total in $B$ which is equivalent to the subspace spanned by $\left\{u_{\alpha}\right\}$ being dense in $B$.

For $f \varepsilon B, H(f, F ; r)$ defines a linear functional with norm $N(F ; r)$, so if $F \varepsilon B^{0}$

$$
\gamma(f)=\lim _{r \rightarrow(1, \ldots, 1)} H(f, F ; r)
$$

aefines an element of $B^{*}$ corresponding to $F$. The passage from $F$ to $\gamma$ given by (4.5) defines an operator $\Lambda: B^{0} \rightarrow B^{*}$ where $\Lambda(F)=\gamma$ 。

THEOREM 4.3. Let $B$ be a Banach space of type $A_{4}$. Then $\Lambda$ is a linear operator,

$$
\begin{gathered}
\Gamma \Lambda(F)=F \text {, for } F \in B^{0} \text {, and } \\
\left\|\Lambda(F)|| \leq N(F) \leq A_{4}(B)|| \Lambda(F)\right\| \text {, for } F \in B^{0} \text {. }
\end{gathered}
$$

Thus $\Lambda$ defınes an injective mapping, with bounded inverse, of $B^{0}$ onto a subspace of $B^{\star}$. PROOF: Let $F \in B^{0}, \Lambda(F)=\gamma$ and $\Gamma(Y)=G$. Then $\gamma_{\alpha}(G)=\gamma\left(u_{\alpha}\right)=\lim _{r>(l, \ldots, 1)} H\left(u_{\alpha}, F ; r\right)=\lim _{r \rightarrow(l, \ldots, l)} \gamma_{\alpha}(F) r^{\alpha}=\gamma_{\alpha}(F)$, and so $\Gamma(\Lambda(F))=F$. Also, since $|H(f, F ; r)| \leq|| f|||| F||$ it follows that $|\gamma(f)| \leq|| f|||| F||$ where $\gamma=\Lambda(F)$. Thus, from (4.6)

$$
\|F||=|| \Gamma(\Lambda(F))\| \leq|| \Gamma||\|\Lambda(F)\|=A_{4}(B)|| \Lambda(F) \| .
$$

If the space $B$ satisfies an additional axiom, viz.,

$$
P_{5}: \text { If } f \in B \text { then } T_{r} f \in B \text { and } \lim _{r \rightarrow(1, \ldots, 1)}\left\|T_{r} f-f\right\|=0 \text {, }
$$

then $\mathrm{B}^{*}, \mathrm{~B}^{\prime}$ and $\mathrm{B}^{0}$ turn out to be isometrically isomorphic.

THEOREM 4.4. Let $B$ be a Banach space of type $A_{4}$ also satisfying $P_{5} \cdot$ Then

$$
\begin{aligned}
& B^{0}=B^{\prime} \\
& \Lambda \text { is bijective and isometric, and } \\
& \Lambda^{-1}=\Gamma .
\end{aligned}
$$

PROOF : BY (1.19) H(T $f, F ; \rho)=H\left(T_{\rho} f, F ; r\right)$ and so

$$
H(f, F ; r)-H(f, F ; \rho)=H\left(f-T_{\rho} f, F ; r\right)+H\left(T_{r} f-T_{\rho} f, F ; \rho\right)+H(T \rho f-f, F ; \rho) 。
$$

Therefore, if $\mathrm{f} \varepsilon \mathrm{B}$ and $\mathrm{F} \varepsilon \mathrm{B}^{\prime}$

$$
|H(f, F ; r)-H(f, F ; \rho)| \leq\left[2|| f-T_{\rho} f||+|| T_{r} f-T_{\rho} f||\right] N(F),
$$

and by $\mathrm{P}_{5}$

$$
\lim _{r, \rho \rightarrow(1, \ldots, 1)}|H(f, F ; r)-H(f, F ; \rho)|=0,
$$

so $F \in B^{0}$. Since $B^{0} \subset B^{\prime}$ already, this means $B^{\prime}=B^{0}$. 
From $P_{5}, \lim _{r \rightarrow(1, \ldots, 1)}|| T_{r} f||=|| f||$. If $\left|w_{i}\right|=r_{i}<1, i=1, \ldots, n$, and $T_{w} f$ is analytic in $w$ then $\left\|T_{w} f|=| \mid T_{x} f\right\|$ by $P_{3}$. Hence from the maximum modulus theorem for functions with values in a Banach space (see [3], p. 211) ||$T_{r} f||$ is a nondecreasing continuous function of $r$. This yields $A_{4}(B)=1$ and by Theorem 4.3 .1 is isometric. Now suppose $f \in B$ and $\gamma \in B^{*}$, Then

$$
\begin{aligned}
{[\Lambda(\Gamma(\gamma))](f) } & =\lim _{r \rightarrow(1, \ldots, 1)} H(f, \Gamma(\gamma) ; r) \\
& =\lim _{r \rightarrow(1, \ldots, 1)} \gamma\left(T_{r} f\right)=\gamma(f) .
\end{aligned}
$$

As a corollary of Theorem 4.4 the following theorem provides a representation of $B^{*}$ in terms of the Hadamard product

THEOREM 4.5. Under the hypotheses of Theorem 4.4 every linear functional $\gamma \varepsilon B^{*}$ is representable in the form

$$
\begin{gathered}
\gamma(f)=\lim _{r \rightarrow(1, \ldots, 1)} H(f, F ; r)= \\
\lim _{r \rightarrow(1, \ldots, 1)}\left(\frac{1}{2 \pi i}\right)^{n} \int_{T_{\varepsilon_{1}}} \times \ldots \times T_{\varepsilon_{n}} f\left(\xi_{1}, \ldots . \xi_{n}\right) F\left(\frac{r}{\xi_{1}}, \ldots, \frac{r_{n}}{\xi_{n}}\right) \frac{d \xi_{1}}{\xi_{1}} \ldots \frac{d \xi_{n}}{\xi_{n}},
\end{gathered}
$$

where, $0<\varepsilon_{i}<1,0<r_{i}<\xi_{i}<1, i=1, \ldots, n$ and $F \varepsilon B^{0}$. F uniquely determines and is uniquely determined by $\gamma$ and ||$\gamma||=N(F)$.

Equation (4.16) can be expressed in terms of real integrals using (1.21).

\section{REFERENCES}

1. TAYLOR, A. E. Banach Spaces of Functions Analytic in The Unit Circle, I, II, Studia. Math. 11 (1950), pp. 145-170; Studia. Math. 12 (1951), pp. 25-50.

2. GRIMM, L. J. and HALL, L. M. An Alternative Theorem for Singular Differential Systems, J. Diff. Equa. 18 (1975), 411-422.

3. HALL, L. M. A Characterization of The Cokernel of a Singular Fredholm Differential Operator, J. Diff. Equa. 24 (1977), 1-7.

4. HALL, L. M. Regular Singular Differential Equations Whose Conjugate Equation Has Polynomial Solutions, Siam J. Math. Anal. $\underline{8}$ (1977), 778-784.

5. TAYLOR, A. E. Introduction To Functional Analysis, John Wiley and Sons, Inc., New York, 1967. 


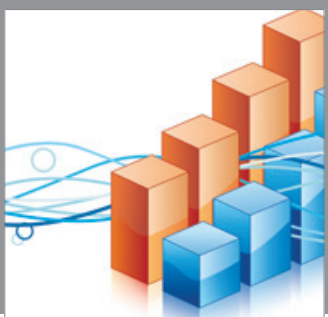

Advances in

Operations Research

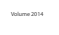

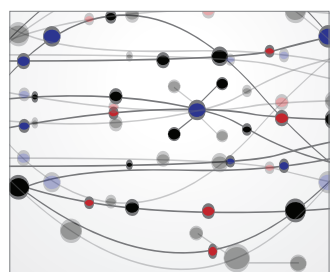

\section{The Scientific} World Journal
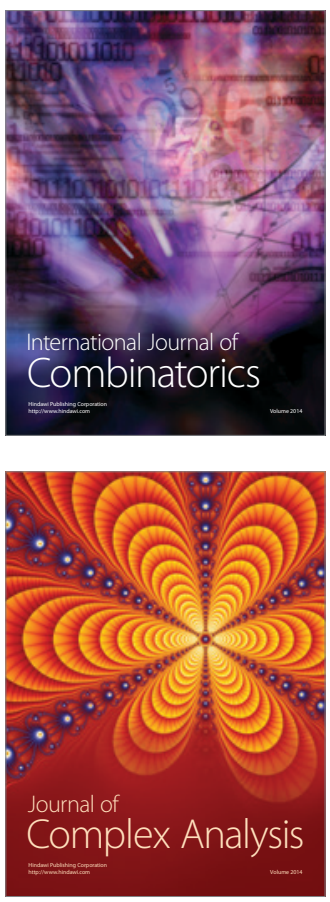

International Journal of

Mathematics and

Mathematical

Sciences
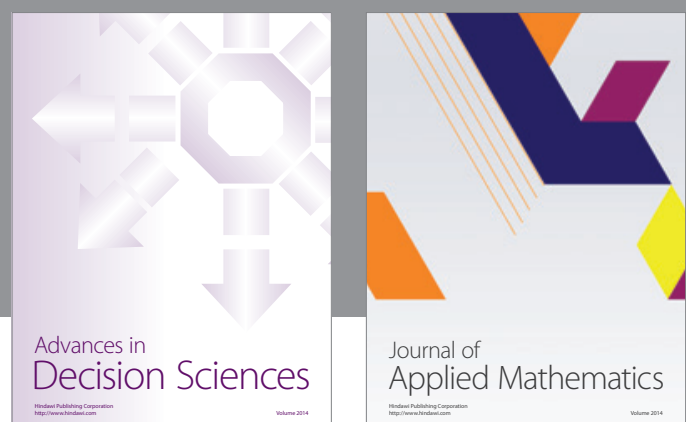

Journal of

Applied Mathematics
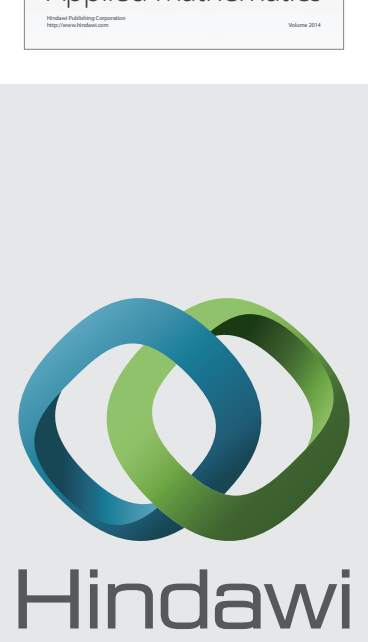

Submit your manuscripts at http://www.hindawi.com
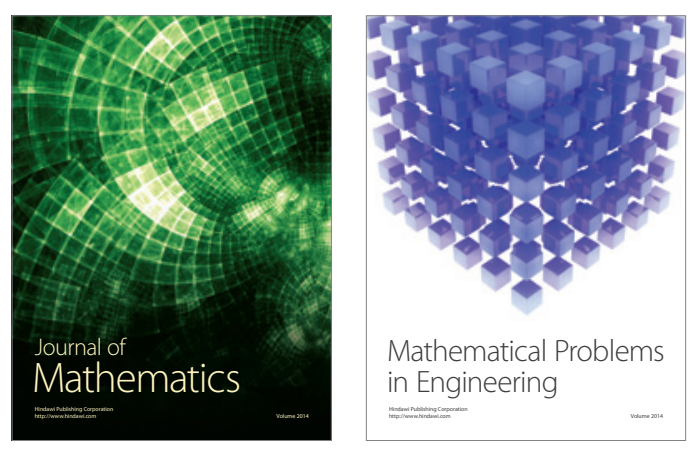

Mathematical Problems in Engineering
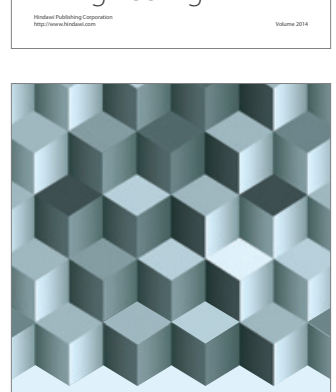

Journal of

Function Spaces
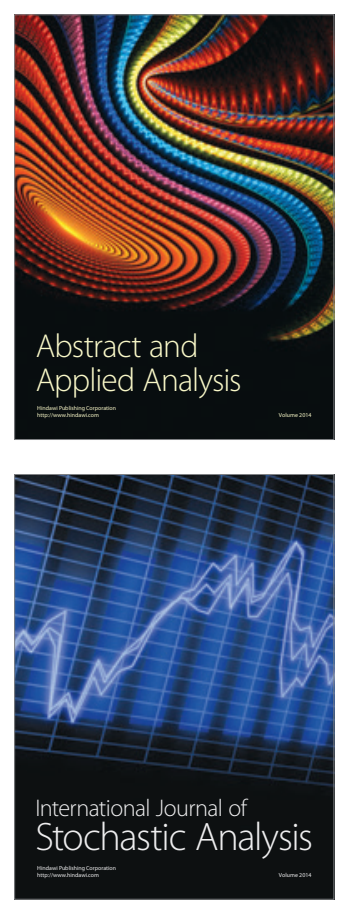

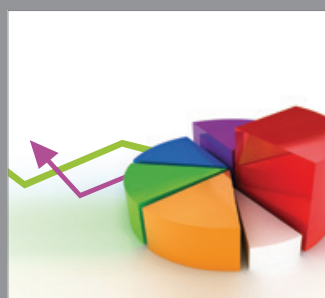

ournal of

Probability and Statistics

Promensencen
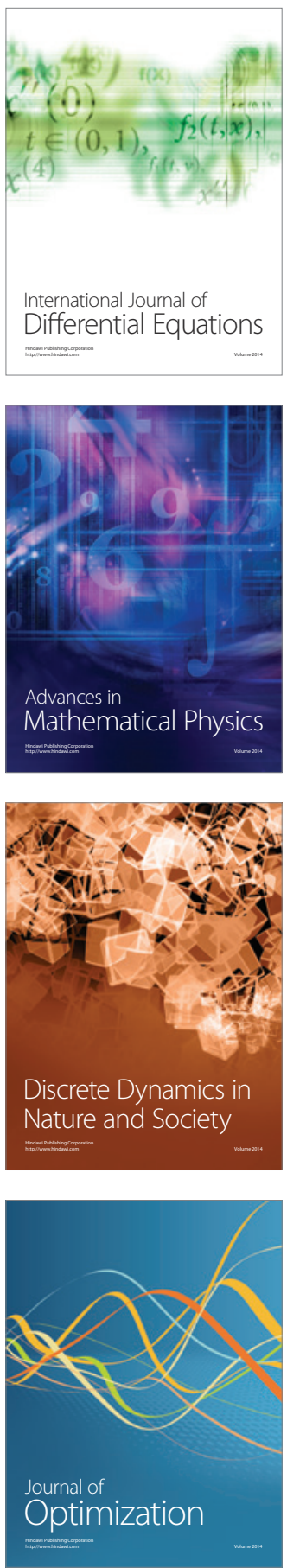